\title{
Development of novel Vitamin B12 fortified yogurts using isolated and microencapsulated Vitamin B12
}

\section{Abstract}

Adults aged $>50$ years, which make up to $40 \%$ of the European population, are vulnerable to low vitamin B12 (B12) status due to age-related factors that impair digestion and absorption of protein-bound B12 from natural food sources. Older adults are recommended to meeting their dietary B12 intake through the consumption of B12-fortified foods or supplements because these products contain free B12. B12 seems most bioavailable from milk products compared to other food sources, showcasing dairy as a potential vehicle for B12 food fortification. Yogurt is a versatile, popular dairy product, making it a promising food vehicle for B12 fortification to enhance the availability of B12-fortified foods for population groups at risk of B12 deficiency. With the overall goal to develop a novel, B12-fortified dairy product, the objective of this project was to compare the shelf-life stability of different chemical forms of B12 added to yogurt either in isolated or in encapsulated form. For both fortification strategies, we compared methylcobalamin (MeCB), a naturally-occurring B12 form, and cyanocobalamin (CnCB), the synthetic form of B12. Encapsulated microparticles were created by spray-drying a maize starch-derived polymeric material (EUDRAGUARD Natural ${ }^{\circledR}$ ) with $1 \%(w / V)$ MeCB or $\mathrm{CnCB}$. The release of $\mathrm{B} 12$ from encapsulated microparticles was confirmed by in vitro simulated gastrointestinal digestion; maximum B12 release (103\% recovery) was achieved under conditions simulating the small intestine, where B12 is absorbed in the human body. Yogurts were produced by incubating whole milk $\left(3.25 \%\right.$ milk fat) with yogurt starter bacteria at $43^{\circ} \mathrm{C}$ overnight; after fermentation

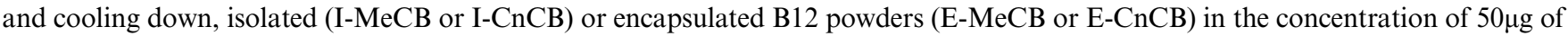
$\mathrm{B} 12 / 175 \mathrm{~g}$ of yogurt were added, creating stirred yogurt. Yogurts were stored $a 4^{\circ} \mathrm{C}$ for eight weeks, with intermittent sampling for B12 stability testing using RIDASCREEN immunoassay. For CnCB-fortified yogurts, I-CnCB and E-CnCB had similar B12 concen-

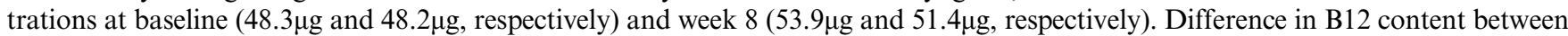
I-CnCB and E-CnCB was $<5 \%$ for both time-points. For $\mathrm{MeCB}$, similar concentrations were found for I-MeCB $(64.1 \mu \mathrm{g})$ and $\mathrm{E}-\mathrm{MeCB}(65.0 \mu \mathrm{g})$ at baseline; however, at week $8, \mathrm{E}-\mathrm{MeCB}$ remained stable $(69.8 \mu \mathrm{g})$ while a pronounced decrease in $\mathrm{B} 12$ was observed with $\mathrm{I}-\mathrm{MeCB}(33.3 \mu \mathrm{g})$. At week $8, \mathrm{I}-\mathrm{MeCB} \mathrm{B} 12$ concentrations were $52 \%$ lower than $\mathrm{E}-\mathrm{MeCB}$. In conclusion, $\mathrm{CnCB}$ was shown to be the more stable fortificant throughout shelf-life. Encapsulation techniques are a viable option to increase $\mathrm{MeCB}$ stability in fortified yogurts.

\section{Conflict of Interest}

There is no conflict of interest. 